\title{
Myocarditis: A clinical perspective
}

\author{
JOANNA PEASE, DO \\ CHARLES C. TSAI, MD \\ GEORGE A. WILLIAMS, MD \\ MARTIN W. SCHWARZE, DO
}

Myocarditis can have many diverse presentations ranging from ventricular arrhythmias to congestive heart failure, secondary to dilated cardiomyopathy. The recent resurgence of endomyocardial biopsy has greatly enhanced our ability to diagnose this intriguing entity. The biopsy has shown that unexplained arrhythmias have a $15 \%$ to $29 \%$ incidence of myocarditis, while those with an idiopathic congestive cardiomyopathy have a biopsyproved range of $9 \%$ to $63 \%$. The cause of this process has, at times, been blamed on viruses, but definite cause-and-effect relationships are mostly conjecture and anecdotal. The current role of immunosuppressive therapy in myocarditis is potentially promising, and may alter the subsequent course of the illness.

\footnotetext{
From the department of internal medicine/cardiology. Metropolitan Medical Center, West, St Louis, and the departments of pathology and internal medicine/cardiology, St Louis University School of Medicine, St Louis, Mo. Dr Pease is presently in private practice in Detroit, Mich; Dr Tsai is associate professor of medicine, St Louis University School of Medicine; Dr Williams is associate professor of medicine, St Louis University School of Medicine and chief of cardiology, St Louis VA Medical Center; and Dr Schwarze is assistant clinical professor of medicine, St Louis University School of Medicine, chief of staff, Barnes-St Peters Hospital, St Peters, Mo, and staff physician, Metropolitan Medical Center.

Reprint requests to Martin W. Schwarze, DO, Metropolitan Medical Center, West, 530 Des Peres Rd, St Louis, MO 63131.
}

With the increasing use of percutaneous endomyocardial biopsy (EMB), acute lymphocytic myocarditis is now being diagnosed even when typical clinical findings are absent. This improved diagnostic ability has shown that myocarditis can cause not only acute myocardial dysfunction, but also chronic heart ailments, such as persistent ventricular arrhythmias or idiopathic congestive cardiomyopathy (ICCM). However, many questions concerning myocarditis still need to be answered. The pathogenesis and immunology of the disease are currently not well delineated, and the role of immunosuppressive medications in the treatment of the disease is still being debated. Therefore, the purpose of this article is to review the current knowledge about myocarditis and address those issues that are still controversial. Two case reports are presented to illustrate some of the many ways myocarditis can present, and will demonstrate one case where immunosuppression was found to be of prolonged benefit, while the other showed only transient improvement.

\section{Report of cases \\ Case 1}

In November 1982, a 35-year-old man was seen in the outpatient department for evaluation of palpitations. The patient had an irregular heart beat 2 years earlier, but did not become concerned until he developed prolonged episodes of dizziness that were associated with palpitations. The patient denied chest discomfort, shortness of breath, parox- 
ysmal nocturnal dyspnea, orthopnea, or syncope. He was receiving no medications, and his medical history was remarkable only for malaria. The patient denied smoking or alcohol consumption. His mother and sister both had palpitations.

On physical examination, the patient was in no distress. His blood pressure was $122 / 90 \mathrm{~mm} \mathrm{Hg}$. There was no jugular venous distention. The carotid upstrokes were adequate, and there were no bruits. The lungs were clear. The cardiac examination revealed the point of maximal impulse (PMI) to be mildly displaced to the left, but the heart sounds were normal and there were no murmurs, rubs, or gallops. Results of the abdominal examination were normal. The peripheral pulses were full and there was no peripheral edema.

A noninvasive cardiac evaluation was performed. Results of a 24-hour Holter monitoring showed an average of nine premature ventricular contractions per hour, multiple ventricular couplets, and multiple episodes of ventricular tachycardia, with several eight-beat runs with a ventricular rate of 150 beats. Results of an echocardiogram were unremarkable. An exercise tolerance test failed to provoke ventricular arrhythmias.

In December 1982, a cardiac catheterization showed normal coronary arteries and only mild apical hypokinesis of the left ventricle. An endomyocardial biopsy specimen was taken from the apex of the right ventricle and demonstrated a grade I myocarditis. Immunosuppressive treatment was therefore instituted using prednisone $(100 \mathrm{mg})$ and azathioprine ( $125 \mathrm{mg} /$ day). The arrhythmias were also treated with atenolol and procainamide, and improved over the course of 1 year.

In January 1984, after a year of therapy, another biopsy specimen was obtained from the right ventricular apex, guided by two-dimensional echocardiography. This time the biopsy specimen had a perfectly normal histologic appearance with no evidence of interstitial fibrosis. The dosage of prednisone and azathioprine was then tapered. In January 1985 , a biopsy specimen was obtained, again from the right ventricle. Again there was no evidence of active myocarditis and only mild interstitial fibrosis was seen. The patient's arrhythmias also remained significantly improved. At the time of his last evaluation, while receiving quinidine gluconate, the patient had only rare couplets and no runs of ventricular tachycardia.

\section{Case 2}

A 24-year-old woman was seen by the cardiology service at St Louis (Mo) University in June 1982 because of progressive shortness of breath, which was consistent with congestive heart failure. These symptoms had been present for 9 years prior to this office visit, but, in recent months, had become progressively worse, requiring her to seek additional care.

Ten years earlier she had syncopal episodes secondary to complete heart block, with intermittent episodes of ventricular tachycardia. She was treated with a permanent ventricular demand pacemaker, with alleviation of the symptoms. The patient did not smoke or drink alcohol. Her family history was negative for cardiovascular disease.

Results of a physical examination in 1982 revealed an alert, well-oriented woman who was of short stature and had mild respiratory distress. The blood pressure was $120 / 80 \mathrm{~mm} \mathrm{Hg}$. The lungs were clear to auscultation and percussion. The cardiac examination revealed the PMI to be diffuse in the fifth intercostal space, along the anterior axillary line. The first heart sound was intact, the second heart sound was paradoxically split. There were no murmurs, friction rubs, or gallop rhythms noted.

In 1982, results of a Holter monitor examination demonstrated a ventricular paced rhythm with ventricular ectopic beats, without couplets or ventricular tachycardia. Results of an echocardiogram were consistent with a dilated cardiomyopathy.

The patient required progressively higher doses of diuretic therapy and unloading therapy. Her symptoms continued to worsen, and an endomyocardial biopsy subsequently demonstrated grade 2 myocarditis. Azathioprine (Immuran) and prednisone therapy was begun, and during the course of the next year her condition improved markedly. She continued to receive digoxin (Lanoxin) and captopril, but had marked reductions in the quantity of diuretic therapy needed. In the fall of 1983 , the patient was seen at the hospital because of an altered mental status associated with a fever. This condition was believed to result from infection and improved with conservative medical therapy. However, at the time of discharge, azathioprine and prednisone therapy was stopped because it was believed that these medications contributed to her recent infectious process. One week following discharge, the patient died suddenly.

\section{Classifications of myocarditis}

At the present time, we use the following classification. Grade 0 represents an essentially normal myocardium. Grade 1 myocarditis is diagnosed when lymphocytic infiltration is pre- 
sent, but no active cell destruction is seen in any section (Fig 1 ). Grade 2 active lymphocytic myocarditis is diagnosed when lymphocytic infiltration and active cell destruction are seen histologically (Fig 2). Grade 3 myocarditis is diagnosed when lymphocytic and white blood cell infiltration are present, cell destruction is more extensive, and immunoglobulin deposition is present as indicated by immunofluorescent techniques (Fig 3). In grade 4 myocarditis, these same changes are present with widespread acute cell inflammation and active cell destruction (Fig 4).

\section{Clinical features}

Myocarditis can have many diverse clinical presentations. Before the widespread use of endomyocardial biopsy, myocarditis was diagnosed only on clinical grounds. Patients were thought to have myocarditis if they had no coronary artery disease and if there was a history of at least two of the following: a preceding viral infection, sudden onset of congestive heart failure, pleuritic chest pain, fever, or pericardial friction rub. Patients also had to have characteristic electrocardiographic changes such as diffuse ST- or T-wave changes, low QRS voltage, A-V conduction defects, intraventricular conduction defects, or arrhythmias. ${ }^{1}$ However, as illustrated by these case reports, multiple series using endomyocardial biopsies have shown that many cases of myocarditis do not present with the typical symptoms.

The reported incidence of histologically proved myocarditis is between $17 \%$ and $65 \% .^{2,3}$ Myocarditis may be present asymptomatically, as an isolated cause of unexplained ventricular arrhythmias, or as atypical chest pain. Approximately $10 \%$ of routine autopsies and up to $10 \%$ of patients dying of systemic infectious diseases without clinical evidence of cardiac involvement show histologic evidence of myocardial inflammation. ${ }^{4}$ In a study by Strain and associates, ${ }^{5}$ patients with unexplained arrhythmias had a $15 \%$ to $29 \%$ incidence of myocarditis; of those with atypical chest pain, up to $20 \%$ have documented inflammation. ${ }^{6} \mathrm{~Pa}$ tients with myocarditis and normal left ven- tricular function often complain of atypical chest pain.

One of the most well-documented manifestations of myocarditis is idiopathic congestive cardiomyopathy (ICCM). Idiopathic congestive cardiomyopathy is a primary disease of heart muscle that is characterized by cardiomegaly and dilated ventricles. The incidence of ICCM is at least 3 to 5 cases per 100,000 population per year, ${ }^{7}$ and has classically been considered a disease of unknown cause. However, with the increasing use of EMB, there is now growing evidence that myocardial inflammation may be one causative factor. The number of patients with ICCM that have myocarditis by EMB ranges from $9 \%$ to $63 \% .1,3,8$ One reason for such a wide range of figures relating myocarditis to ICCM is that investigators have used differing classification systems in the histologic diagnosis of myocarditis. ${ }^{1,9,10}$ Edwards considered that at least five lymphocytes per high-power field had to be present to make a reliable diagnosis of myocarditis. ${ }^{11}$ In contrast, Shanes and associates ${ }^{12}$ and Zee-Cheng and associates, ${ }^{2}$ suggested that even minimal lymphocytic infiltration was clinically responsive to immunosuppression if myofibrillar degeneration or necrosis were also present.

Discrepancies in the findings of the incidence of myocarditis in ICCM could also be due to sampling errors. Inflammation is often focal, so that unless multiple samples are analyzed, the small amount of tissue obtained by EMB might not contain involved areas. Although it is clear that myocarditis can be associated with ICCM, the true prevalence of myocarditis in patients with ICCM still needs to be determined.

\section{Role of viruses}

Although the cause or causes of myocarditis may be multifactorial, much of our present information has come from the study of viral myocarditis. Viruses are the most commonly known factors related to myocarditis, with coxsackievirus causing at least half of the cases. ${ }^{2,13}$ Coxsackievirus myocarditis is not infrequent. Epidemics of the virus have resulted in a $12 \%$ incidence of cardiac involvement. 


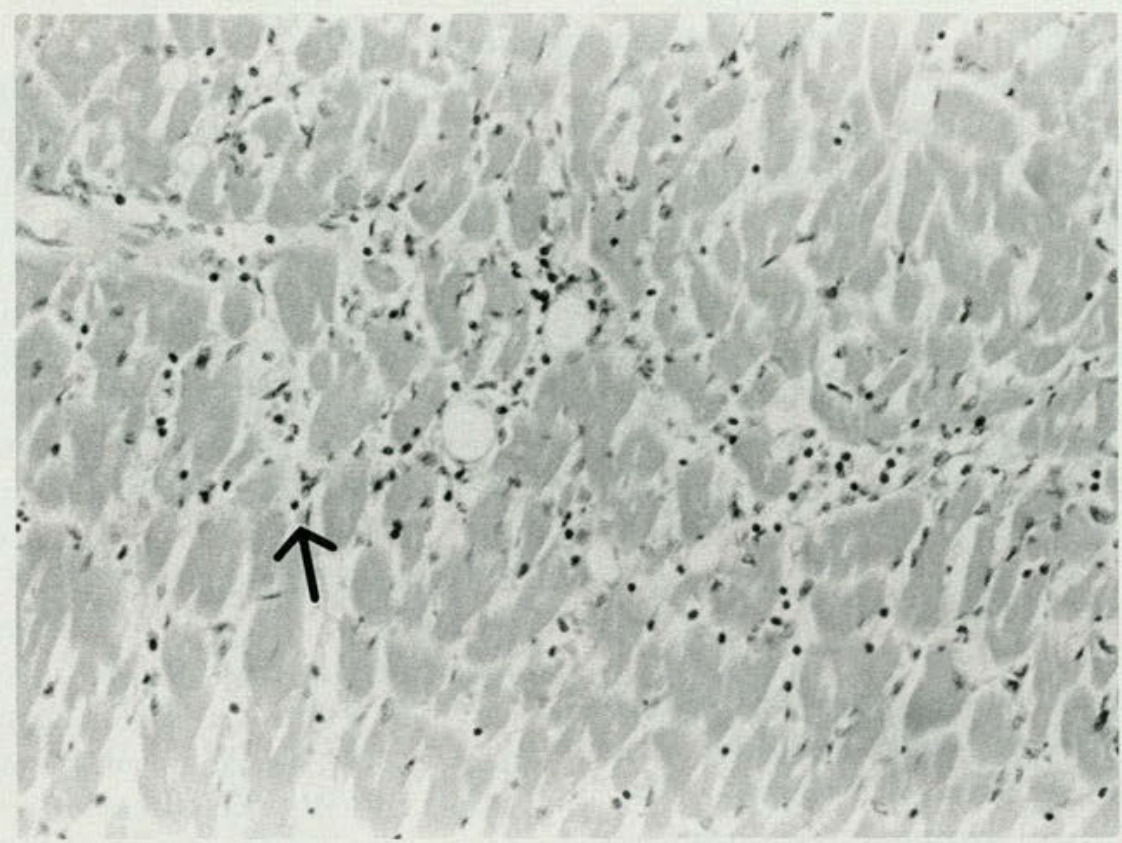

Figure 1. Grade 1 myocarditis. In this low-power (original magnification $\times 160)$ photomicrograph, scattered lymphocytic infiltration can be seen, represented by the small black dots alongside the sarcomeres (arrow).

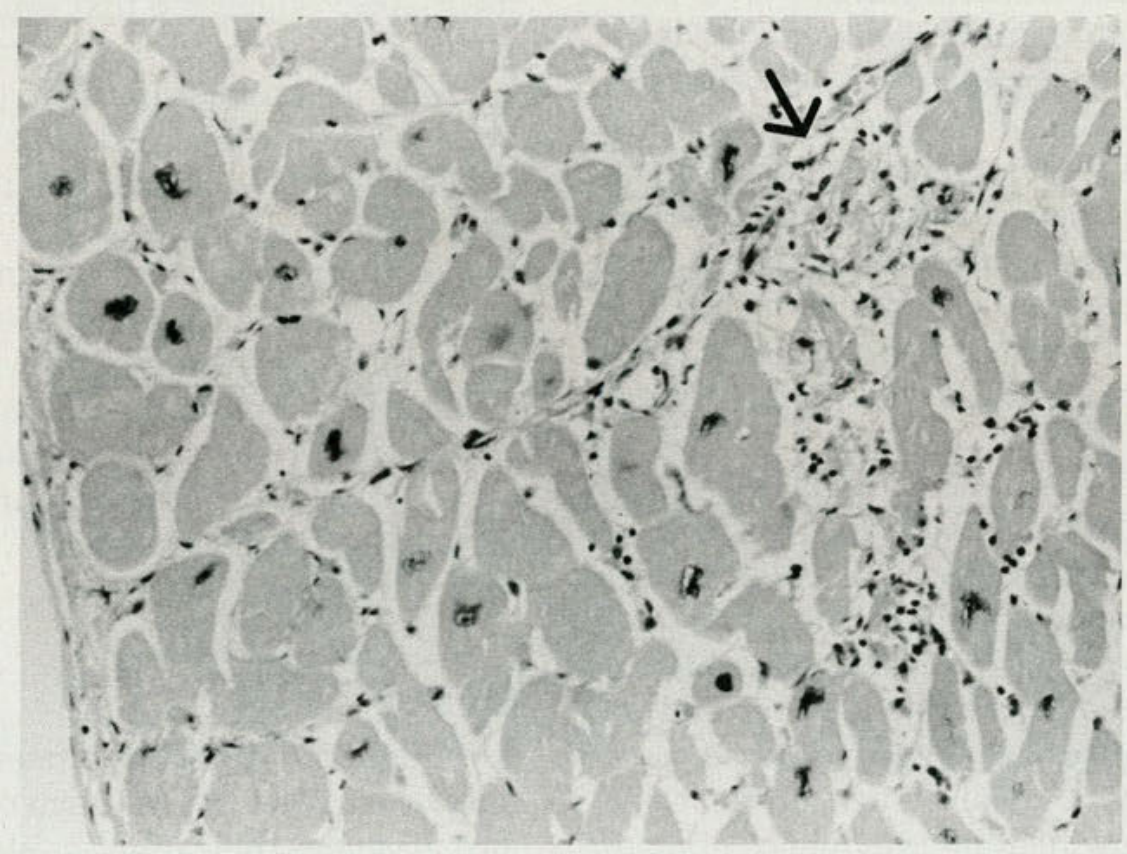

Figure 2. Grade 2 myocarditis. Subendocardial thickening with scattered lymphocytic infiltration can ben seen (left side of photograph), along with a focus of lymphocytes surrounding a degenerating cell represented by the blurred myocardial edges and multiple patches of severe inflammatory infiltration (arrow). 


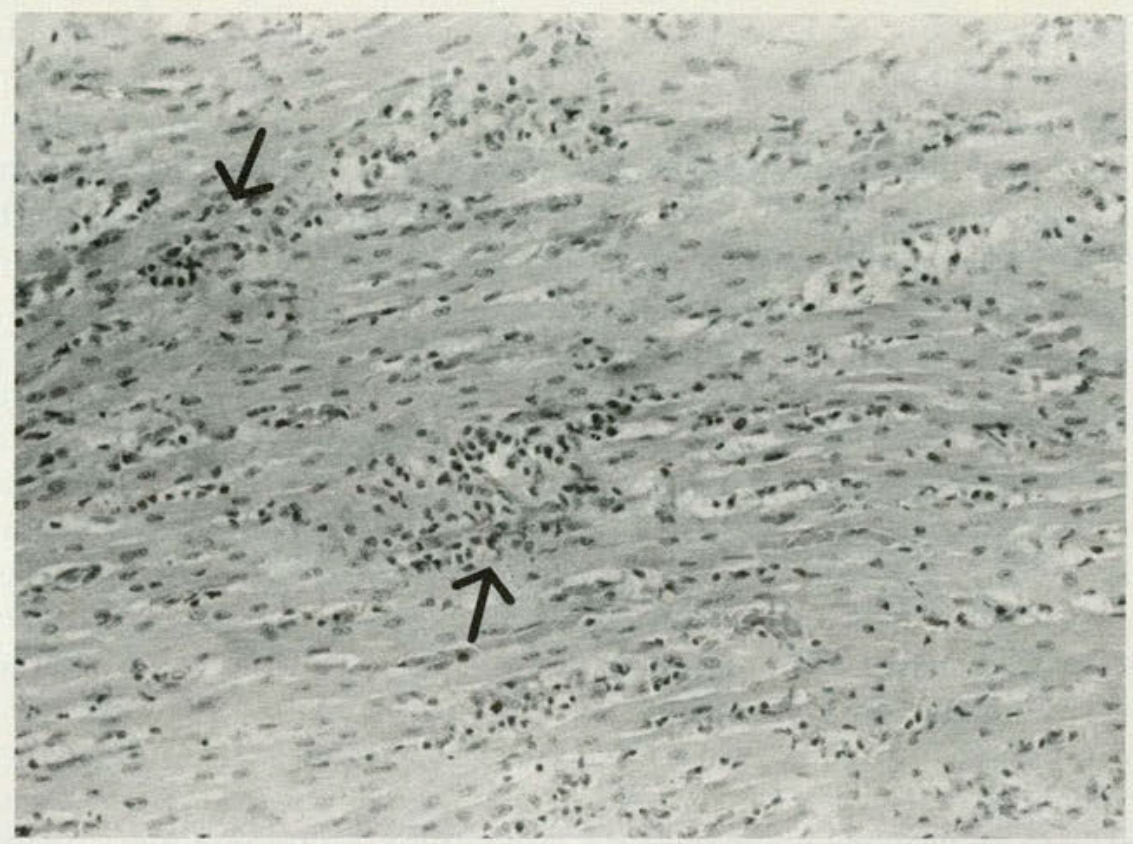

Figure 3. Grade 3 myocarditis. Diffuse lymphocytic infiltration is seen, with several areas of degenerating cells represented by the blurred myocardial edges and multiple patches of severe inflammatory infiltration (arrows).

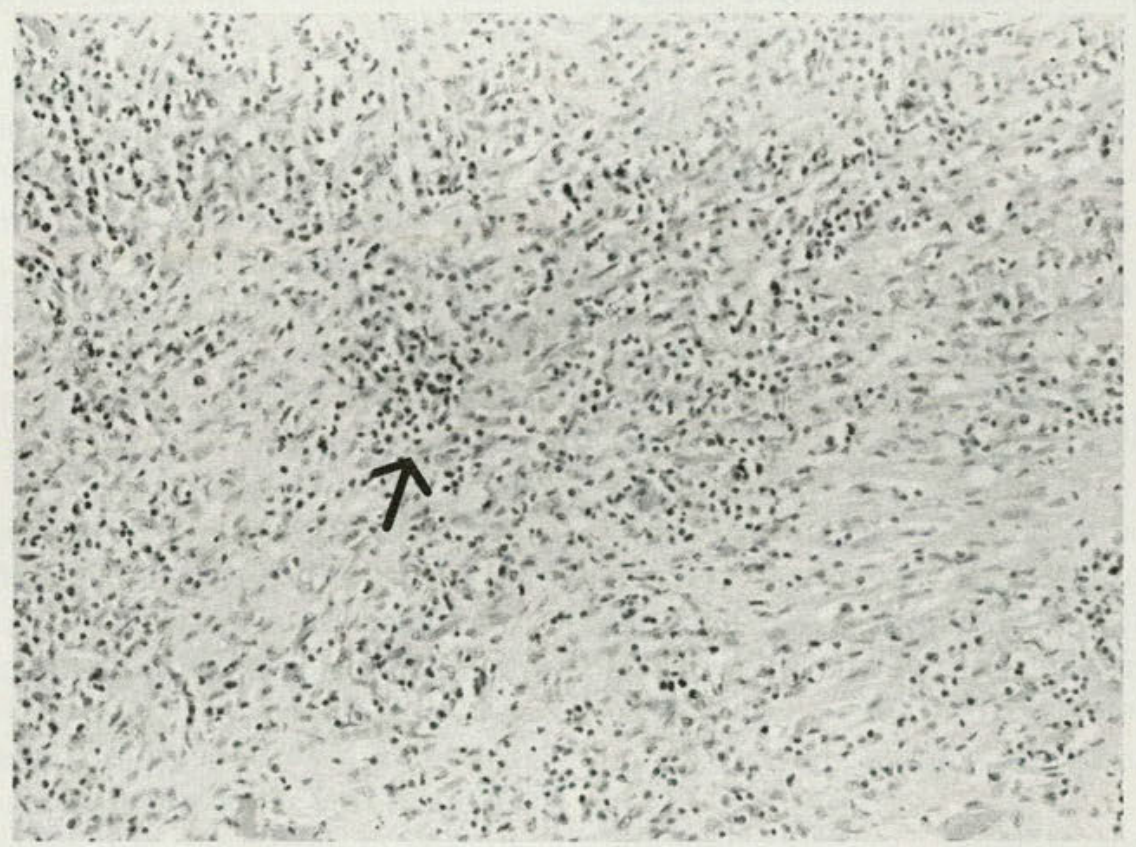

Figure 4. Grade 4 myocarditis. Diffuse inflammatory cell infiltration is present, with almost total obliteration of myocytes in the center (arrow). 
In adolescents and adults, males are most often affected, ${ }^{2,13}$ and there is evidence in animal models that several factors may worsen the severity of the disease. These factors include hypoxia, malnutrition, exercise, pregnancy, radiation, and trauma. ${ }^{14}$ Most patients with coxsackievirus B myocarditis recover completely; there is a reported $50 \%$ to $80 \%$ complete recovery rate and only a $0 \%$ to $5 \%$ death rate. ${ }^{15}$

In some patients with acute coxsackievirus B myocarditis, chronic congestive cardiomyopathy may develop. The possibility that viruses can, in fact, cause ICCM has been difficult to demonstrate because viruses can be cultured only in acute cases of the disease, ${ }^{11,13}$ and therefore cannot usually be cultured from patients with ICCM. However, long-term follow-up studies of patients with documented acute coxsackievirus B myocarditis have now shown a $15 \%$ to $20 \%$ incidence of persistent congestive heart failure. ${ }^{16-18}$ The finding that increased titers of neutralizing antibody to coxsackievirus B is present in patients with new onset (ie, duration of less than 1 year) congestive cardiomyopathy also supports the role of the virus in the pathogenesis of ICCM. ${ }^{19}$ In addition, viral myocarditis may cause persistence of chest pain and cardiac arrhythmias after the acute infection has subsided. ${ }^{11}$

\section{Immunologic findings}

The actual mechanism by which a virus damages the heart has been studied most extensively in mice. Viremia occurs 24 to 72 hours after infection with subsequent viral attachment to myocardial receptor sites. Viral replication then occurs in cell cytoplasm. ${ }^{20}$ Replication peaks after 3 to 4 days when host-defense mechanisms become operative. Viral titers cannot be detected after 7 to 10 days, and there is no myocardial antigen detectable after 14 days. $^{21}$

The murine host-defense capability depends on several different immunologic mechanisms. In the first 5 to 10 days, large numbers of monocytes infiltrate the myocardium. Neutralizing antibody also appears. This antibody can abort infection in vitro, and has been shown to prevent infection after active immunization in a model using encephalomyocarditis virus. ${ }^{22}$ Monocytes are also essential in preventing viral dissemination. In steroid-treated mice, severe lesions develop in spite of normal neutralizing antibody levels, because the number of monocytes present in the tissues is markedly reduced. ${ }^{23}$ Thus, both monocytes and neutralizing antibody play a critical role in controlling viral replication.

In contrast to the protective function of antibody and monocytes, $T$ cells enhance the amount of myocardial damage, and may be the cause for persistent myocardial dysfunction after the acute infection subsides. Much less cardiac necrosis and significantly fewer deaths occur in coxsackievirus-infected mice that are made deficient in $\mathrm{T}$ cells by thymectomy or by treatment with antithymocyte globulin. ${ }^{24}$ The actual mechanism of T-cell cytotoxicity is complex. Cytotoxic T cells are produced in the spleen. In the early response ( 3 to 5 days), there is no viral specificity, and both infected and uninfected myocardial cells are lysed, that is, both virus-specific and autoreactive nonvirus-specific cells (natural killer cells) are produced. $^{25}$

Interestingly, the response in male and female mice differs. Females show persistence of the ability to lyse uninfected cells, whereas cytotoxicity in males after 1 week causes lysis of only infected fibers. ${ }^{26}$ In addition, males have higher viral titers with an increased magnitude of T-cell response and an increase in mortality. ${ }^{26}$ These findings are relevant because, in humans, myocarditis more often affects males than females. ${ }^{27}$

The immunologic mechanisms in humans are less well defined. Abnormalities in cellmediated immunity have been found. There is a decreased ability of concanavalin A-activated T cells to suppress autologous cells in the mixed lymphocyte response. ${ }^{27}$ Also, defective natural killer cell activity has been found in $50 \%$ of one group of patients with ICCM. ${ }^{16}$ Subtyping cells in the inflammatory infiltrate of the myocardium has shown the presence of monocytes, T cells, and B cells. The exact role of these cells in the pathogenesis of myocarditis is still unclear, however, and further study is needed in this area. 
The humoral mechanisms causing myocardial inflammation and permanent damage in humans are also not well delineated. There is controversy over whether the presence of heartreactive antibody in the serum of patients with ICCM represents a primary causative mechanism or a secondary response to previous myocardial damage. Maisch and colleagues ${ }^{28}$ found a statistically significant increase in musclespecific antimyolemmal antibodies (AMLA) in patients with congestive cardiomyopathy that had had previous perimyocarditis, and found that the degree of cytolytic activity against karyocytes was directly proportional to the AMLA titer. Other investigators, however, have found no correlation between heart-reactive antibodies and the degree of heart damage. Heart-reactive antibodies are present in the serum of $12 \%$ to $28 \%$ of patients with ICCM, but have been found to be more prevalent in patients with other types of immune cardiac disease such as rheumatoid arthritis or lupus erythematosus, ${ }^{29}$ therefore implying that humoral factors play only a secondary role.

\section{Treatment}

The standard treatment for myocarditis has been to provide supportive care and to minimize the risk factors. Because viral myocarditis is exacerbated in mice by exercise, malnourishment, or hypoxia, adequate rest, nutrition, and oxygen have been recommended. Despite the fact that congestive heart failure responds to standard therapy, and because of the realization that immunologic mechanisms have significant impact on the pathogenesis and course of the disease, immunosuppressive medications are now being used more often in documented myocarditis with deterioration. It must be remembered that during acute viral myocarditis a much higher mortality may occur when steroids are given. ${ }^{30}$

Early case reports of immunosuppression in humans either showed dramatic success or dramatic failure with steroid therapy., ${ }^{1,2,17}$ However, more recent reports using immunosuppression and EMB for diagnosis and follow-up have shown some encouraging results. In sev- eral small uncontrolled clinical trials using from 3 to 34 patients with left ventricular dysfunction due to myocarditis, $40 \%$ to $100 \%$ of the patients showed either improvement or stabilization of cardiac function after immunosuppressive therapy was instituted..$^{2,4,5,10,12,17}$ In those studies in which serial endomyocardial biopsies were performed, the amount of myocardial inflammation decreased substantially when there was clinical improvement ${ }^{2,9,10}$; however, there is one study that did not show a correlation. ${ }^{18}$ Improvement was also seen in a small group of patients with only ventricular arrhythmias as a manifestation of their myocarditis. Further evidence suggesting a beneficial effect of immunosuppressive medications is that the tapering of therapy caused reexacerbation of disease in selected patients. Reinstitution of immunosuppression then resulted in renewed control of the disease..$^{2,5,8,12,28,30}$ In some cases, the clinical exacerbations and remissions also correlated with the degree of inflammation on EMB. ${ }^{2,12}$

In spite of these encouraging results, the studies conducted so far are not conclusive. First, none of the studies was controlled and the number of patients studied was small. Establishing controls is essential because spontaneous recovery may occur. As mentioned previously, the likelihood of complete spontaneous recovery in viral myocarditis is high. Even in patients with significant chronic left ventricular dysfunction due to myocarditis, a spontaneous recovery rate of up to $33 \%$ has been reported ${ }^{8}$ Second, there is some evidence that the beneficial effects of immunosuppressives may be only transient. ${ }^{17}$ Third, it may be that only certain groups of patients with myocarditis are candidates for immunosuppressive therapy. $\mathrm{Pa}$ tients with chronic or low-grade inflammation respond well to therapy, whereas those with acute or rapidly progressive disease have a steadily downward course whether or not treatment is instituted. ${ }^{2,10}$ However, even this relationship between severity of inflammation and efficiency of therapy is contested. In a retrospective study by Dec and associates, ${ }^{8} 44 \%$ of patients treated with immunosuppressives and $33 \%$ of those untreated improved regardless of the severity of inflammation. 


\section{Conclusion}

In summary, there is still a lot to be known about myocarditis. Certainly, since the widespread use of EMB, the multiple manifestations of myocarditis such as left ventricular dysfunction, unexplained ventricular arrhythmias, and chest pain have been better defined. However, the exact incidence of myocarditis in patients with chronic cardiomyopathy is debated mostly because a uniform classification system for the diagnosis of myocarditis has still not been developed. Although epidemiologic and serologic data suggest that viruses cause many cases of myocarditis and ICCM, the immunologic mechanism by which myocardial damage is produced still is under investigation. Last, although several studies have shown promising results, the benefit of immunosuppressive therapy will continue to be uncertain until evidence from prospective and controlled trials becomes available.

We are indebted to Wendy K. Anzilotti, Mary E. Streif, and Patti Niemeyer for their assistance in the preparation of this manuscript.

1. Koontz CH, Ray CG: The role of Coxsackie group B virus infection in sporadic myopericarditis. Am Heart $J$ 1971;82:750758.

2. Zee-Cheng CS, Tsai CC, Palmer DC, et al: High incidence of myocarditis by endomyocardial biopsy in patients with idiopathic congestive cardiomyopathy. J Am Coll Cardiol 1984;3:6370.

3. Nippoldt TB, Edwards WD, Holmes DR, et al: Right ventricular endomyocardial biopsy: Clinicopathologic correlates in 100 consecutive patients. Mayo Clin Proc 1982;57:407-418.

4. Abelmann WH: Myocarditis. $N$ Engl J Med 1966;275:832834.

5. Strain JE, Grose RM, Factor SM, et al: Results of endomyocardial biopsy in patients with spontaneous ventricular tachycardia but without apparent structural heart disease. Circulation 1983;68:1171-1181.

6. Miklozek CL, Kingsley EM, Schoen FJ, et al: Normal noninvasive left ventricular systolic function in biopsy proven myocarditis. Circulation 1983;68(suppl 111):28.

7. Torp A: Incidence of congestive cardiomyopathy. Postgrad Med J 1978;54:435-437.

8. Dec WG, Palacios IF, Fallon JT, et al: Active myocarditis in the spectrum of acute dilated cardiomyopathies: Clinical features, histologic correlates, and clinical outcome. N Engl J Med 1985;312:885-890.

9. Edwards WD, Holmes DR, Reeder GS: Diagnosis of active lymphocytic myocarditis by endomyocardial biopsy: Quantitative criteria by light microscopy. Mayo Clin Proc 1982;57:419425.
10. Fenoglio JJ, Ursell PC, Kellogg CF, et al: Diagnosis and classification of myocarditis by endomyocardial biopsy. $N \mathrm{Engl}$ J Med 1983;308:12-18.

11. Wilson FM, Miranda Q, Chason J, et al: Residual pathologic changes following Coxsackie A and B myopericarditis. Am $J$ Pathol 1969;55:253-265.

12. Shanes JG, Krone RJ, Tsai CC, et al: Mild myocardial inflammation presenting as congestive cardiomyopathy responsive to immunosuppression. Am Heart $J$ 1984;107:798-801.

13. Woodruff JF: Viral myocarditis: A review. Am J Pathol 1980;101:427.

14. Abelmann WH: Virus and the heart. Circulation 1971;44:950.

15. Sinani G, Dekate M, Rao CP: Heart disease caused by Coxsackie virus B infection. Br Heart $J$ 1975;37:819.

16. Anderson JL, Carlquist JF, Hammond EH: Deficient natural killer cell activity in patients with idiopathic dilated cardiomyopathy. Lancet 1982;2:1124-1127.

17. Williams GA, Schwarze M, Kennedy HL, et al: Treatment of chronic active myocarditis with immunosuppressive therapy. $J$ Am Coll Cardiol 1984;3:521.

18. Strain JE, Grose R, Hirsch CL, et al: Discordance of clinical and histological response to therapy in chronic myocarditis. $J$ Am Coll Cardiol 1984;3:520.

19. Woodruff JF: Lack of correlation between neutralizing antibody production and suppression of Coxsackie virus B-3 replication in target organs: Evidence for involvement of mononuclear inflammatory cells in host defense. J Immunol 1979;123:3136.

20. Rabin ER, Hassan SA, Jenson AB, et al: Coxsackie virus B3 myocarditis in mice. Am J Pathol 1964;44:775-797.

21. Woodruff JF: The influence of quantitated post-weaning undernutrition on Coxsackie virus B3 infection of adult mice: II. Alteration of host defense mechanisms. J Infect Dis 1970;121:164181.

22. Matsumori A, Crumpacker C, Abelmann WH, et al: Virus vaccine and passive immunization for the prevention of viral myocarditis in mice. Circulation 1983;68(suppl 111):338.

23. Kilbourne ED, Wilson CB, Perrier D: The induction of gross myocardial lesions by a Coxsackie (pleurodynia) virus and cortisone. J Clin Invest 1956;35:362-370.

24. Woodruff JF, Woodruff JJ: Involvement of T-lymphocytes in the pathogenesis of Coxsackie virus B3 heart disease. J Immunol 1974;113:1726-1734.

25. Wong CY, Woodruff JJ, Woodruff JF: Generation of Coxsackie virus B3 infection: I. Model and viral specificity. $J$ Immunol 1977;118:1159-1164.

26. Wong CY, Woodruff JJ, Woodruff JF: Generation of cytotoxic T lymphocytes during Coxsackie virus B3 infection: III. Role of sex. J Immunol 1977;119:591-597.

27. Fowles RE, Bieber CP, Stinson EB: Defective in vitro suppressor cell function in idiopathic congestive cardiomyopathy. Circulation 1979;59:483-491.

28. Maisch B, Deeg P, Liebau G, et al: Diagnostic relevance of humoral and cytotoxic immune reactions in primary and secondary dilated cardiomyopathy. Am J Cardiol 1983;52:10721078.

29. Das SK, Cassidy JF, Petty RE: Antibodies against heart muscle and nuclear constituents in cardiomyopathy. Am Heart J 1972;83:159-166.

30. Aretz HT, Chapman C, Fallon JT: Morphologic and immunologic findings in patients with clinically suspected myocarditis. Circulation 1983;68(suppl 111):27. 


\section{Help The Ad Council curb drunk driving.}

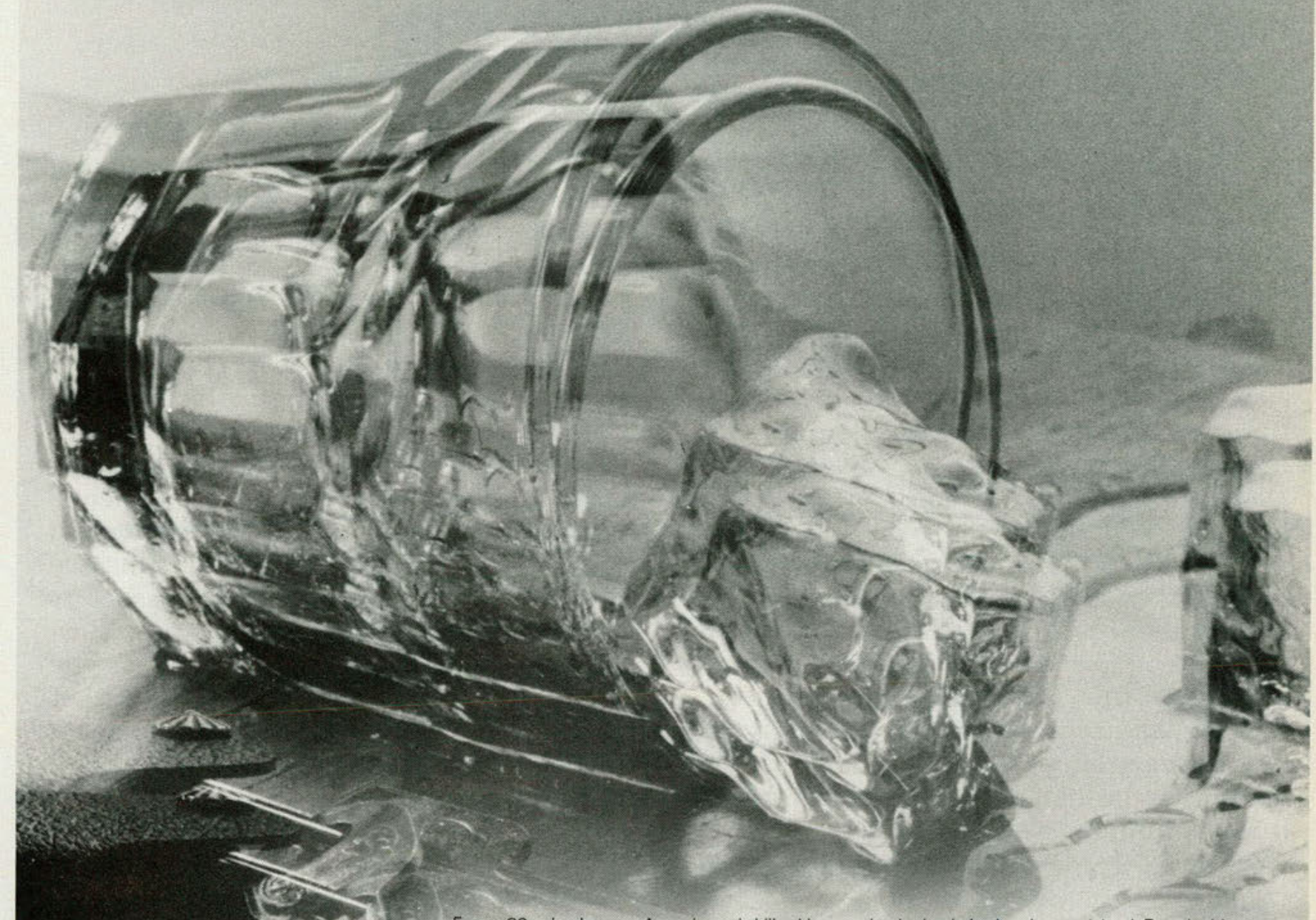

Every 20 minutes, an American is killed in an alcohol-related auto accident. Drunk drivers are responsible for half of all deaths on our roads and highways.

The Advertising Council began a campaign in 1983 to curb this devastating problem, which-counting deaths, injuries and property loss - costs U.S. taxpavers an estimated $\$ 24$ billion yearly.

To expand the reach and impact of such programs, The Advertising Council needs the financial support of you or your organization. Drunk driving prevention is just one of more than 30 public service campaigns we undertake each year, with the help of agency and media volunteers... and you. Each dollar you invest in the Council translates into \$735 in contributed time and space by the media.

\section{Make a difference. Support The Advertising Council.}

rd like to help

The Advertising Council

The Advertising Council

825 Third Avenue

New York, New York 10022

212.758 .0400 $\square$ Here's my tax-deductible contribution.

$\square$ Please send more information to: 